\title{
Extraction of the beam elastic shape from uncertain FBG strain measurement points
}

\author{
M. Pinto ${ }^{(1)}$, N. Roveri ${ }^{(1)}$, G. Pepe ${ }^{(1)}$, A. Nicoletti ${ }^{(2)}$, G. Balconi ${ }^{(3)}$, A. Carcaterra $^{(1)}$ \\ ${ }^{1}$ Dep. of Mechanical and Aerospace Engineering, Sapienza University of Rome \\ ${ }^{2}$ BASF Italy \\ ${ }^{3}$ SIREG GEOTECH
}

Keywords: Structural health monitoring, Strain measurements, GFRP, FBG, Neural Network Classifier.

\begin{abstract}
Aim of the present paper is the analysis of the strain along the beam that is equipped with Glass Fibers Reinforced Polymers (GFRP) with an embedded set of optical Fiber Bragg Grating sensors (FBG), in the context of a project to equip with these new structural elements an Italian train bridge.

Different problems are attacked, and namely:

(i) during the production process [1] it is difficult to locate precisely the FBG along the reinforcement bar, therefore the following question appears: how can we associate the strain measurements to the points along the bar? Is it possible to create a signal analysis procedure such that this correspondence is found?

(ii) the beam can be inflected and besides the strain at some points, we would like to recover the elastic shape of the deformed beam that is equipped with the reinforcement bars. Which signal processing do we use to determine the shape of the deformed beam in its inflection plane?

(iii) if the beam is spatially inflected, in two orthogonal planes, is it possible to recover the beam spatial elastic shape?

Object of the paper is to answer to these questions.
\end{abstract}

\section{Introduction}

The use of vibration measurements for structural health monitoring has attracted significant research attention during the last three decades [2-4]. Traditional strengthened concrete members, such as beams, are composed of concrete included cement and steel bars reinforcement. In such structures, the main function of concrete is to provide resistance to compressive loads. Steel bars embedded in the concrete, instead, provide resistance to tensile and shear loads. Nevertheless, the problem of corrosion associated with the steel bars reduced its live time. Recent technologies have resulted in alternative reinforcing materials such as GFRP materials that can be embedded in concrete members to satisfy several desired properties.

GFRP represent an attractive opportunity in the field of new concrete constructions as well as in the field of restoration of old ones. Their weight and resistance properties 
provide good chances of replacement of traditional steel reinforcements in some applications [5-7].

FBG sensors allow distributed sensing over significant areas by multiplexing a large number of sensors on a single fiber, which are immune to electromagnetic interference and have compact size. Due to all these advantages, they have been widely used in many applications for the structural health monitoring $[8,9]$.

This project started as a collaboration between the department of Mechanical and Aerospace Engineering of Sapienza University of Rome, BASF Italy Spa and SIREG GEOTECH srl. The main task of such project is to perform a structural restoration on a train bridge, located over Bormida river, in northern Italy. Instead of using traditional steel bars reinforcements, for this structure the previously mentioned GFRP rebars with embedded FBG sensors, will be adopted.

By the use of a main station, suitably implemented, it will be possible to process all the data acquired through the sensors inside the structure, in order to achieve information about the stress state of the bridge to perform a structural health monitoring activity.

\section{Uncertainties in the measurements}

A general difficulty we meet with fiber glass reinforcement bars are related to some uncertainties on the sensors locations. In general, we asked to produce each bar with three embedded optical lines, each equipped with at least one sensor. This configuration, as it comes out from the following considerations, permits to solve the underdeterminacy intrinsically related to: (i) the axial positioning of the sensors on each line along each bar, (ii) the angular positioning of each bar inside the beam.

However, since the radius of each bar is small with respect to the characteristic size of the beam section, and the radius of the circle over which the optical lines are placed is even smaller, we can approximate the nominal optical line positioning on each section as it is identified by the centre of each bar. In this way the only unknown parameters of the sensors placements are related to their axial positions. This implies that, in general, each sensor could be potentially located at an unknown axial position, but in this way, as it is clarified by the following sections, the number of unknowns is too large to be determined. Therefore, we need to reduce the degree of uncertainty of the system. One good point is that of producing bars that host optical line made with FBG sensors placed at given constant distance d. With this, we can resolve the under-determinacy of the system, as it is explained in the following section.

\section{Analytical model for predicting the system deformation}

Task of the proposed method is to estimate the elastic shape of the beam through the measurements of axial deformations in several points along the beam, whose positions are unknown. This method is applied to a beam subjected to bending deformation according to the Euler-Bernoulli hypothesis. The unknown elastic shape of the beam can be expressed in form of an expansion of known eigenfunctions $\phi_{k}(x)$, combined 
through a set of weighting unknown time-dependent coefficients, that are the modal coordinates $A_{k}(t)$. Since the distributed-parameter system has infinitely many vibration modes, the general response is a linear combination from all vibration modes:

$$
w(x, t)=\sum_{k=1}^{\infty} A_{k}(t) \phi_{k}(x)
$$

where $w(x, t)$ is the transverse displacement of the neutral axis (at the point $x$ and the time $t$ ) due to bending.

The axial strain at a certain level $(z)$ from the neutral axis of the beam is simply proportional to the curvature of the beam at that position $(x)$ :

$$
\varepsilon_{i}^{(j)}=-z_{i}^{(j)} \frac{d^{2} w\left(x_{s i}^{(j)}, t\right)}{d x^{2}}=-z_{i}^{(j)} \sum_{k=1}^{\infty} A_{k}(t) \frac{d^{2} \phi_{k}}{d x^{2}}\left(x_{s i}^{(j)}\right)
$$

Where $\varepsilon_{i}^{(j)}$ is the measured strain provided by the FBG sensors and $x_{s i}^{(j)}$ is the longitudinal position of the $i$-th sensor along the $j$-th fiber.

However, if the position $x_{s i}^{(j)}$ are unknown, together with the unknown coefficients $A_{k}(t)$, an undetermined set of conditions is produced which is unsolvable, since the number of unknowns is larger than the number of conditions. Since along the single optical fiber there are several FBG sensors that, due to the product specifications, are equally spaced at a known constant spacing $d$, it is possible to reduce the original number of unknowns $i \times j\left(x_{s i}^{(j)}\right)$ in the only $j$ line offset $x_{s o}^{(j)}$ unknown variables. From this hypothesis, the eq. 2 becomes:

$$
-\frac{\varepsilon_{i}^{(j)}}{z_{i}^{(j)}}=\sum_{k=1}^{\infty} A_{k} \frac{d^{2} \phi_{k}}{d x^{2}}\left(x_{s o}^{(j)}+(i-1) d\right)
$$

The equation system is easily solved: using $N_{B}$ fibres, each carrying $N_{S}$ sensors, the total number of available equations are $N_{e q}=N_{B} \times N_{S}$. The number of unkonwns depends on the number of considered modal coordinates $N=N_{A_{k}}$, indeed we have $N_{B} \times N_{A_{k}}$ and $N_{B}\left(x_{s o}^{(j)}\right)$ number of line offsets, for a total number of unknown variables to identify equal to $N_{u n}=N_{B} \times N+N_{B}$. Such system can be solved through least square if it is satisfied the condition: $N_{e q} \geq N_{u n}$.

The problem of determining the elastic shape of the beam is simultaneously solved, since the solution of the previous system provides also the coefficients $A_{k}$, i.e. through $w(x)=\sum_{k=1}^{\infty} A_{k} \phi_{k}(x)$ one can determine the elastic shape.

For example, assume that the elastic shape is represented only by two coefficients $A_{1} A_{2}(N=2)$ and considering the beam simply supported, we can write the mode shape in harmonic form as $w(x, t)=A_{1}(t) \sin \left(\frac{\pi x}{L}\right)+A_{2}(t) \sin \left(\frac{2 \pi x}{L}\right)$ and an algorithm for the solution of the nonlinear equation eq. (3) can now be set.

Assume we introduce a first guess for the offsets $x_{s o}^{(j)}$, the linear system is defined in matrix form:

$$
\boldsymbol{U}\left(x_{s o}^{(j)}\right) \boldsymbol{A}=\boldsymbol{b} \rightarrow \boldsymbol{A}=\boldsymbol{U}^{+}\left(x_{s o}^{(j)}\right) \boldsymbol{b}
$$


Where $\boldsymbol{b}=\left\{\frac{\varepsilon_{i}^{(j)}(t)}{z_{i}^{(j)}}\right\} \quad$ is the column vector of the acquired strains and $\boldsymbol{U}=$ $\left\{\left(k \frac{\pi}{L}\right)^{2} \sin \left[\frac{k \pi\left(x_{s o}^{(j)}+(i-1) d\right)}{L}\right]\right\}$ is an $N_{S} \times N$ matrix and where $\boldsymbol{U}^{+}$is the pseudo-inverse matrix. The equation error is now introduced:

$$
e=\left\|\boldsymbol{A}-\boldsymbol{U}^{+}\left(x_{s o}^{(j)}\right) \boldsymbol{b}\right\|
$$

Now, fixed an initial guess for the offsets $x_{s o}^{(j)}$, an optimal set of coefficient $\boldsymbol{A}$ is obtained, then the problem is iterated varying $x_{s o}^{(j)}$, to find the minimum point $\bar{x}_{s o}^{(j)}$ so that a suitable error function will be minimised and $\bar{x}_{s o}^{(j)}$ will be the estimated offset for the fiber $j$.

As explained, the error function $e\left(x_{s o}^{(j)}\right)$ defined in eq. (5) depends indeed on the initial guess of the offset. We can set a first guess for $x_{s o}^{(1)}$, for example $x_{s o}^{(1)}=\frac{L}{M}$, where $\mathrm{M}$ is a given integer, thus we obtain an overdetermined set of equations in two unknowns $A_{1} A_{2}$ that can be solved in a least square sense (by using the pseudo-inverse), and evaluate the corresponding error $e\left(x_{s o}^{(j)}\right)$. Now we are able to introduce another error function err, slightly different than eq. (5), which is defined as the mean error between the strains measured by the FBG sensors and the strains estimated by eq. (3), having used the optimal set of coefficients $A_{1} A_{2}$ :

$$
\operatorname{err}\left(x_{\text {so guess }}\right)=\sum_{i=1}^{s} \frac{\varepsilon_{i}-\varepsilon_{i}\left(x_{\text {soguess }}\right)}{s \varepsilon_{i}}
$$

Then we can modify the value, as $x_{s o}^{(1)}=\frac{2 L}{M}$, and solve again in the least square sense for $A_{1} A_{2}$, and compute again the error. Let us proceed in this way evaluating the set of errors for $x_{s o}^{(1)}=\frac{k L}{M}, k=1,2,3, \ldots, M$. The value of $k$ for which the equation error (7) is minimum identifies the estimated value of the offset $x_{s o}^{(1)}$, at which are associated the optimal values for $A_{1} A_{2}$. Therefore, in this way we can associate each of the strain measurement with the related point along the axis, i.e. $x_{s o i}^{(1)}=x_{s o}^{(1)}+(i-1) d$.

The algorithm has been tested applying each time the same load $P$ in different position all over the length of the beam, between 0 and $L$, for a fixed value of the line offset; then the same set of loads have been applied, varying the position of the offset in the range of the maximum error of location, namely between 0 and $d$. The simulations have been carried out considering 39 displacements for the load over the entire length of the beam for a number of fixed offsets equal to 28 ; the results of the test are represented in Fig. 1. 


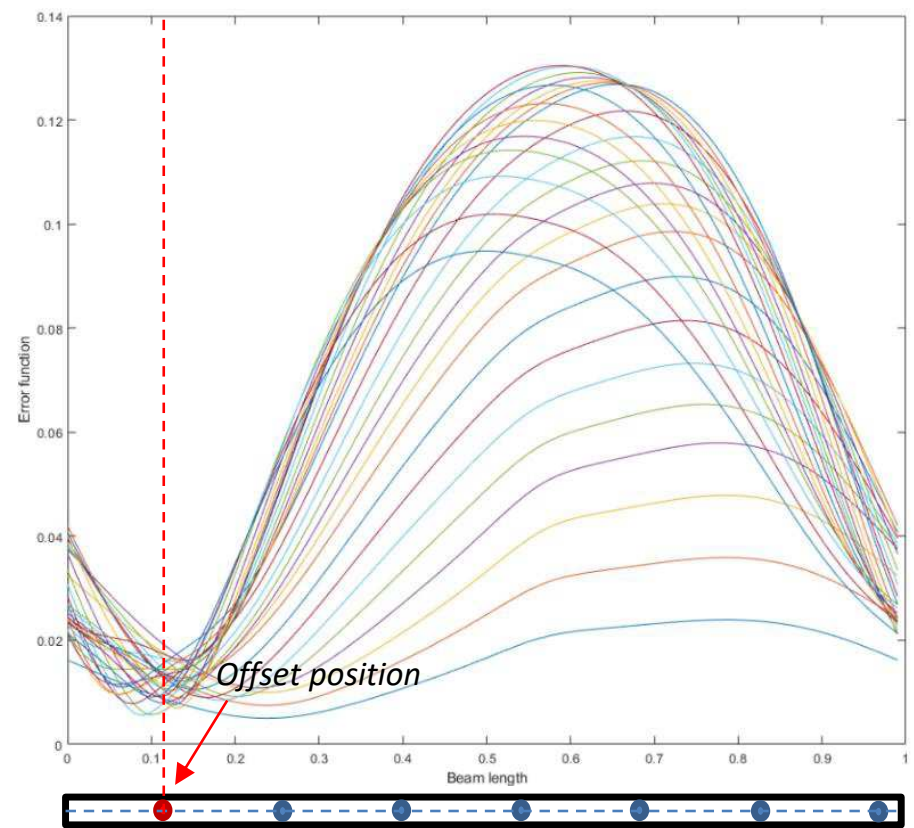

Fig. 1. Simulation results: trend of the $\operatorname{err}\left(x_{\text {soguess }}\right)$ function for one fixed line offset.

Note that, for explanatory purpose, the results reported in the Fig.1 refer to just one fixed value of line offset $x_{s o}$. In this plot, each curve is the error function, eq. (6), related to a single load, applied in a specific position. The error functions have the same trend, specifically all of them have a minimum in the neighbourhood of the actual offset; however among the set of the curves there are some that provide a better estimation of the offset, while other provide larger errors. The estimation error is therefore introduced, namely the relative error between the actual and the estimated offset:

$$
e_{c}=\left|\frac{x_{o s}-x_{o S_{e s t}}}{x_{o s}}\right|
$$

Where, for the sake of notation, the dependence on the load position $x_{p}$, e.g $e_{c}\left(x_{p}\right)$, has been omitted. Over the entire set of obtained curves, only in certain cases we obtain an optimal solution in which the relative error of convergence is close to zero. The reasons behind this behaviour may depend on the generality of the model, which relies only on a few periodic eigenfunctions adopted to model the mechanical system; however this limits the reliability of the algorithm introduced so far.

\section{Data Post processing with an ANN algorithm}

The algorithm previously introduced works indeed under rather general hypothesis, so that it is able to provide an estimate of the flexural deformation without requiring an accurate mechanical model of the structure. As a result, the algorithm can be applied to a wide set of structures, the trade-off is that the accuracy of the offset estimation is 
affected by the actual placement of the offset and of the load that is exciting the structure. For a specific placement of the offset, the proposed technique shows indeed good accuracy for certain load positions, while it is not effective for others. To overcome the problem, an Artificial Neural Network [10] algorithm is implemented, to classify the reliable curves, which provide a good localization of the line offset, from the other curves that, on the contrary, provide wrong estimations.

For each actual placement of the offset and of the load, the input vector that feeds the neural network will be composed by the following features:

1. A set of g-error values $\operatorname{err}\left(x_{\text {so }}\right.$ guess $\left.j\right)$, defined in eq. (6), evaluated on an array of initial guess, $x_{\text {soguess } j}$, uniformly distributed within the interval $[0$, $d]=[0, \Delta x, \ldots, j \Delta x, \ldots,(g-1) \Delta x]$, where $d<L$, being $L$ the length of the structure, with $g=51$;

2. the estimated offset value $x_{o s_{\text {est }}}$;

3. the prominence value of the error function, e. g. $\frac{\operatorname{err}\left(x_{\text {os }} e_{\text {est }}\right)}{\overline{\operatorname{err}}\left(x_{\text {so }_{\text {guess }} j}\right)}$; where $\overline{\operatorname{err}}\left(x_{\text {soguess } j}\right)$ is the mean value of the error.

4. the total number of minima.

To each input vector is associated a target value, which is the relative error $e_{c}$ involved in the estimation of the offset position, previously introduced in eq. (7).

In order to train the net, a dataset is generated by the iteration of the process above, considering $n$-different positions for the offset, uniformly distributed within the interval $[0, \mathrm{~d}]$, and $m$-different positions for the load, uniformly distributed within $[0, \mathrm{~L}]$, with $n=2848$ and $m=191$. To summarize, the generated dataset will contain a total number of records $n \times m=543968$; each record is composed by $g+3=54$ variables, which form the input of a column vector $V$. The target vector $T$ has only one value, which range from 0 to 1 . After the data have been collected and divided, an important step is the data normalisation, in order to make it easier for the ANN training to extract the relevant information. The input and output data is normalized so that they fall into a standard range, typically -1 to 1 . For pattern recognition problem, a sigmoid transfer function is generally used in the output layer of the network, so target values range from -1 to 1 , which represent the asymptotes of the function. However, this tends to cause difficulties for the training algorithm, which tries to saturate the sigmoid function to meet the target value. It is better to assign target values at the point where the second derivative of the sigmoid function is maximum. For the tangent-sigmoid function, this occurs when the net input is -1 and 1 , which corresponds to output values of -0.76 and +0.76 , therefore the targets are set to values of -0.76 and +0.76 instead of -1 and 1 .

\section{$5 \quad$ Numerical Results}

Results of the network testing are reported. In Fig. 2 red circles represent the actual values of the normalised target used for testing, while the blue dots are the predictions made by the developed ANN. In detail, in the lower side of the graph there are the good estimations while in the upper side there are the false cases. Results show good 
performance since the network is able to properly classify the vast majority of the cases: there is an almost perfect fit between the false cases and the output of the net, while for what concerns the true cases, ANN roughly provides a mean value between less (-0.6) and more $(0.8)$ accurate estimations of the expected target, demonstrating that the reliability of the dataset processing performed by the ANN is high. This is evident in the right subplot, in which the same results are reported in terms of probability density: target and ANN predictions have indeed the same distribution trend.

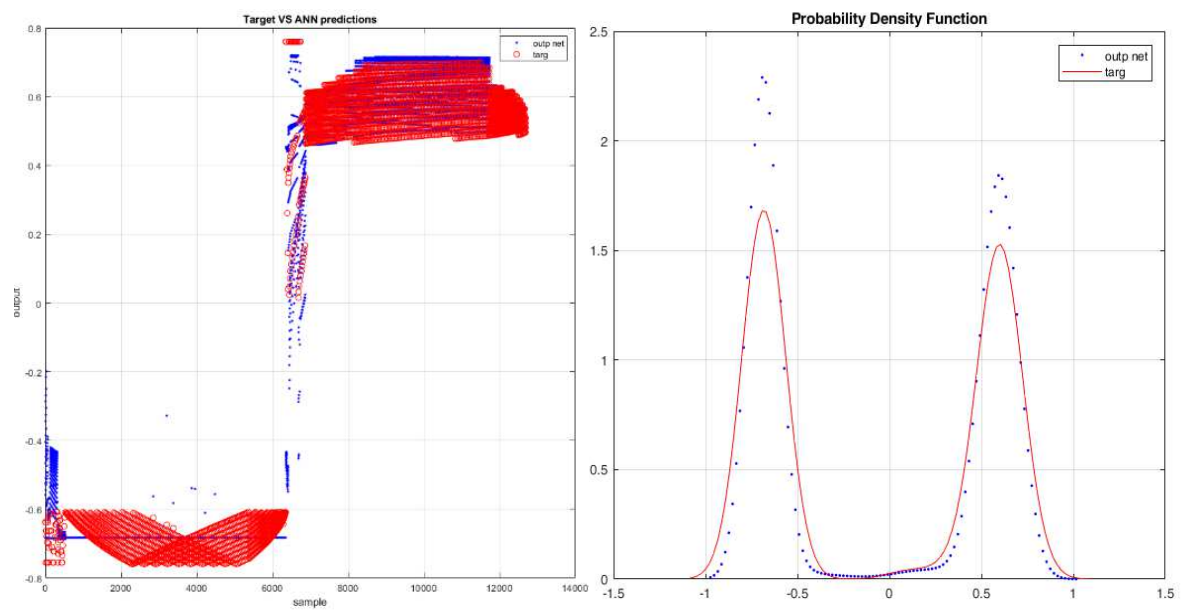

Fig. 2. ANN algorithm results: Network Output VS Target (left), Probability Density Function (right).

\section{Concluding remarks}

The production process does not allow an accurate placements of FBG sensors along a Glass Reinforced Fibers beam. A technique for the identification of the placements of these sensors has been proposed, which relies on two main algorithms. The first algorithm is based on a modal approximation of the elastic shape of the beam, allowing a theorical estimation of its deformation to be compared with the one arising from the strain measurements, which allows a first estimation of the sensors placements. The accuracy of the estimation is however affected by the combination of the actual placement of the sensors and of the load that is exciting the structure. Since the calibration of the measurement apparatus, e.g. the GFRP with embedded FBG sensors inserted into the mechanical/civil structure, can be carried out employing a number of suitable loads, a set of estimations will be at disposal but, among them, only some unknown trials, will provide good results, while other will provide larger errors.

The problem of classify good from bad estimations is solved with a second algorithm, which is based on an Artificial Neural Network, and is able to predict the robustness of the estimation, providing an index that forecasts the error involved in the estimation process, for each position of the exciting load. 
Numerical results shows that the proposed technique is able to locate properly the actual position of the sensors with good accuracy.

The proposed method lays the foundations for future structural health monitoring and damage localization activities in bridge-like structures. The large amount of sensors and vibration measurements allows, with dedicated algorithms [11], the simultaneous identification of both the load characteristics and damage parameters [12].

\section{Acknowledgements}

This work was carried out by the Department of Mechanical and Aerospace Engineering, Sapienza, University of Rome, in collaboration with BASF Italy Spa., and SIREG GEOTECH srl.

\section{References}

1. Zhi Zhou, Thomas W. Graver, Luke Hsu, Jin-ping Ou, Techniques of Advanced FBG sensors: fabrication, demodulation, encapsulation and their application in the structural health monitoring of bridges, Pacific Science Review, vol. 5, 2003, pp.116 121.

2. O.S. Salawu, Detection of structural damage through changes in frequency: a review, Engineering Structures 19 (9) (1997) 718-723.

3. J.N. Yang, P.C. Chang, H. Fujitani, H. Li, B.F. Spencer Jr., Y.L. Xu, F.G. Yuan, Structural control and health monitoring, Proceedings of International Conference on Advances and New Challenges in Earthquake Engineering Research 3 (2002) 9-14.

4. J.W. Lee, J.D. Kim, C.B. Yun, J.H. Yi, J.M. Shim, Health-monitoring method for bridges under ordinary traffic loadings, Journal of Sound and Vibration 257 (2) (2002) 247-264.

5. M. Kemp, D. Blowes Concrete Reinforcement and Glass Fibre Reinforced Polymer Queensland Roads Edition, no. 11 (2011), pp. 40-48.

6. V.R. PatilExperimental Study of Behavior of RCC Beam by Replacing Steel Bars with Glass Fiber Reinforced Polymer and Carbon Reinforced Fiber Polymer (GFRP) (2014).

7. M. Kemp, D. Blowes, Concrete Reinforcement and Glass Fibre Reinforced Polymer, Queensland Roads Edition, no. 11 (2011), pp. 40-48.

8. B. Glisic, D. Inaudi, Fibre Optic Methods for Structural Health Monitoring, Wiley, New York, 2007.

9. F. Vestroni,J. Ciambella, F. Dell'Isola, S. Vidoli, Damage detection with auxiliary subsystems, CIMTEC 2008 - in: Proceedings of Third International Conference on Smart Materials, Structures and Systems - Emboding Intelligence in Structures and Integrated Systems, vol. 56, pp. 401-413.

10. Neural Network Design (2nd Edition) (Inglese) Copertina flessibile - 1 set 2014 di Martin T Hagan (Autore), Howard B Demuth (Autore), Mark H Beale (Autore), Orlando De Jesús (Autore).

11. N. Roveri, A.Carcaterra, A.Sestieri, Real-time monitoring of railway infrastructures using fibre Bragg grating sensors - Mechanical Systems and Signal Processing 60-61(2015)1428.

12. N. Roveri, A.Carcaterra, Unsupervised identification of damage and load characteristics in time-varying systems - Continuum Mech. Thermodyn. (2015) 27:531-550. 